\title{
Errata
}

\section{Transmission Coefficients for Light Projectiles}

\author{
K.H.N. Murthy ${ }^{\star}$, S.K. Gupta, and A. Chatterjee \\ Nuclear Physics Division, Bhabha Atomic Research Centre, Bombay, India
}

Z. Phys. A - Atoms and Nuclei 305, 73 (1982)

Equation (14) of this paper contains an error of sign in the second term. It should read,

$T_{l}=N_{1} f(l)-N_{2} d f(l) / d l$

* DAE Senior Research Fellow, Department of Physics, Mysore University, Mysore - 570006, India
K.H.N. Murthy

S.K. Gupta

A. Chatterjee

Nuclear Physics Division

Bhabha Atomic Research Centre

Van-de-Graaff-Laboratory

Trombay

Bombay -400085

India

\section{An Extension of RPA Theory to Strongly-Interacting Systems}

J.M.C. Chen and J.W. Clark

McDonnell Center for the Space Sciences and Department of Physics,

Washington University, St. Louis, Missouri, USA

D.G. Sandler

Department of Physics, University of Illinois, Urbana, Illinois, USA

Z. Phys. A - Atoms and Nuclei 305, 223 (1982)

The phrase "since $z_{m}=1+O(1 / A)$ for all $m$ under consideration here.", appearing a few lines above (4.20), is incorrect. We thank E. Krotscheck for calling our attention to this fact. The indicated phrase should be replaced by "since $z_{p h p^{\prime} h^{\prime}}=z_{p h} z_{p^{\prime} h^{\prime}}$ $+O(1 / A)$." The latter property allows a common factor $z_{p h}>0$ to be removed from each term of (4.14), (4.17), and the remaining $z$ factors $z_{p^{\prime} h^{\prime}}$ (or $z_{x \beta}$ ) may be absorbed into the definitions of $C$ and $C^{*}$ (see [1]). Such a reinterpretation of $C, C^{*}$ will in fact prove convenient in future formal developments [2].

In addition, the line just below (5.8) should be modified to read: "where $\eta$ is a positive infinitesimal in the continuum problem but may be taken zero for a discrete spectrum."

\section{References}

1. Clark, JW.: The many-body problem, Jastrow correlations versus Brueckner theory. Lecture Notes in Physics. Guardiola, R. Ros, J. (eds.), Vol. 138, p. 184. Berlin, Heidelberg, New York: Springer 1981

2. Sandler, D.G., Krotscheck, E., Clark, J.W.: (to be published)

J.M.C. Chen

J.W. Clark

McDonnell Center for the Space Sciences

and Department of Physics

Washington University

St. Louis, MO 63130

USA

D.G. Sandler

Department of Physics

University of Illinois

Urbana, IL 61801

USA

Responsible for advertisements: E. Lückernann, Kurfürstendamm 237, D-1000 Berlin 15, Telephone: (030) 8821031, Telex: 01-85411 - Springer-Verlag Berlin - Heidelberg New York. - Printers: Universitätsdruckerei H. Stürtz AG, Würburg - Printed in Gemany - C Springer-Verlag GmbH \& Co. KG Berlin Heidelberg 1982 